\title{
Martin representation and Relative Fatou Theorem for fractional Laplacian with a gradient perturbation
}

\author{
Piotr Graczyk • Tomasz Jakubowski • \\ Tomasz Luks
}

Received: 23 April 2012 / Accepted: 20 December 2012 / Published online: 8 January 2013

C The Author(s) 2012. This article is published with open access at Springerlink.com

\begin{abstract}
Let $L=\Delta^{\alpha / 2}+b \cdot \nabla$ with $\alpha \in(1,2)$. We prove the Martin representation and the Relative Fatou Theorem for non-negative singular $L$-harmonic functions on $\mathcal{C}^{1,1}$ bounded open sets.
\end{abstract}

Keywords Gradient perturbation · Fractional Laplacian · Ornstein-Uhlenbeck stable process $\cdot$ Martin representation $\cdot$ Relative Fatou Theorem

Mathematics Subject Classification (2010) $\quad 60 \mathrm{~J} 50 \cdot 60 \mathrm{~J} 75 \cdot 60 \mathrm{~J} 45 \cdot 31 \mathrm{~B} 25$

\section{Introduction and Preliminaries}

\subsection{Motivations}

Analysis of harmonic functions related to fractional powers $\Delta^{\alpha / 2}$ of the Laplace operator is an important topic, intensely developed in recent years, also for

This research was partially supported by ANR-09-BLAN-0084-01 and grants MNiSW N N201 397137, MNiSW N N201 422539.

P. Graczyk · T. Luks

LAREMA, Université d'Angers, 2 Bd Lavoisier, 49045 Angers Cedex 1, France

e-mail: Piotr.Graczyk@univ-angers.fr

T. Luks

e-mail: tomasz.luks@univ-angers.fr

T. Jakubowski $(\bowtie)$

Institute of Mathematics, Wrocław University of Technology,

Wyb. Wyspiańskiego 27, 50-370 Wrocław, Poland

e-mail: tomasz.jakubowski@pwr.wroc.pl 
perturbations of the operator $\Delta^{\alpha / 2}$, see, e.g., $[2,7,11,14,16,25,30,40]$ and references therein.

From the probabilistic point of view, stable stochastic processes with gradient perturbations on $\mathbb{R}^{d}, d \geq 2$, i.e. with the infinitesimal generator

$$
L=\Delta^{\alpha / 2}+b \cdot \nabla,
$$

where $\alpha \in(0,2)$, constitute an important class of jump processes, intensely studied in recent years. Their most celebrated case are the Ornstein-Uhlenbeck stable processes with $b(x)=\lambda x, \lambda \in \mathbb{R}$. They have important physical and financial applications and form a part of Lévy-driven Ornstein-Uhlenbeck processes, cf. [13,43].

The motivations of this paper were to:

(i) establish the theory of the Martin representation for singular $L$-harmonic nonnegative functions

(ii) study boundary limit properties of $L$-harmonic functions and to obtain a Relative Fatou Theorem for them

(iii) develop the theory of Hardy spaces of $L$-harmonic functions.

The topics (i) and (ii) are addressed in this article and the subject (iii) in a forthcoming paper.

All these topics are fundamental for the knowledge of $L$-harmonic functions. The topics (i) and (ii) are well developed for fractional Laplacians. The Martin representation was established in this case in $[6,11,21,41,45]$, see also [37] for a general setting of Markov processes. The Relative Fatou Theorem was proved for $\alpha$-harmonic functions on $\mathcal{C}^{1,1}$ sets in [8], on Lipschitz sets in [39], and on the so-called $\kappa$-fat sets in [32], see also the survey [7, Chapter 3]. Furthermore, some important variants of stable processes such as relativistic, censored and truncated stable processes were studied from the point of view of topics (i) and (ii), see [17, 18,33,35] and [7, Section 3.4]. Nevertheless, the methods of these extensions do not apply to the operator $L$ of the form (1). Let us notice that all our results are also true for Ornstein-Uhlenbeck stable processes.

On the other hand, Martin representations and boundary properties of harmonic functions were widely studied in the case of diffusion operators. The boundary behavior of harmonic functions for the classical Laplacian $\Delta=\sum_{i=1}^{d} \partial^{2} / \partial x_{i}^{2}$ and Lipschitz domains was treated by Hunt and Wheeden in a fundamental and already classical paper [26]. Let us cite e.g. [1,3-5,24,31,46,47,49] for other results on $\Delta$, and $[15,22,23,38,42,44,48]$ for its various generalizations.

Let us mention that the methods of this article give also interesting new results for operators different from $L$. In the case of Laplacians with gradient perturbations, i.e. $\alpha=2$, we get new perturbation formulas for the Green function and the Martin and Poisson kernels (Sect. 3.4).

The potential theory of stable stochastic processes with gradient perturbations was started in the Ornstein-Uhlenbeck case by Jakubowski $[29,30]$. Next, in the general context of gradient perturbations and $\alpha>1$, with a function $b$ from the Kato class $\mathcal{K}_{d}^{\alpha-1}$ it was developed by Bogdan and Jakubowski $[9,10]$ and by Chen et al. [19]. Our work is a natural continuation of the research presented in [10]. 


\subsection{Preliminaries}

In this subsection we recall for the convenience of the reader the basic definitions and results used in the paper. They can be found e.g. in the monography [7] and in the paper [10].

In what follows, $\mathbb{R}^{d}$ denotes the Euclidean space of dimension $d \geq 2, d y$ stands for the Lebesgue measure on $\mathbb{R}^{d}$. Without further mention we will only consider Borelian sets, measures and functions in $\mathbb{R}^{d}$. By $x \cdot y$ we denote the Euclidean scalar product of $x, y \in \mathbb{R}^{d}$. Writing $f \approx g$ we mean that there is a constant $C>0$ such that $C^{-1} g \leq f \leq C g$. As usual, $a \wedge b=\min (a, b)$ and $a \vee b=\max (a, b)$. We let $B(x, r)=\left\{y \in \mathbb{R}^{d}:|x-y|<r\right\}$. For $U \subset \mathbb{R}^{d}$ we denote

$$
\delta_{U}(x)=\operatorname{dist}\left(x, U^{c}\right),
$$

the distance to the complement of $U$.

A nonempty open set $D \subset \mathbb{R}^{d}$ is of class $\mathcal{C}^{1,1}$ at scale $r>0$ if for every $Q \in \partial D$ there are balls $B\left(x^{\prime}, r\right) \subset D$ and $B\left(x^{\prime \prime}, r\right) \subset D^{c}$ tangent at $Q$. If $D$ is $\mathcal{C}^{1,1}$ at some unspecified scale (hence also at all smaller scales), then we simply say that $D$ is $\mathcal{C}^{1,1}$.

The potential theory objects related to the operator $L$ defined in (1) will be denoted with a tilde , while those related to the operator $\Delta^{\alpha / 2}$ will be denoted without it.

Let $p_{D}(t, x, y)$ and $\tilde{p}_{D}(t, x, y)$ be the heat kernels on $D$ of $\Delta^{\alpha / 2}$ and $L$ respectively. Then

$$
\tilde{G}_{D}(x, y)=\int_{0}^{\infty} \tilde{p}_{D}(t, x, y) d t, \quad x, y \in \mathbb{R}^{d}
$$

is the Green function of $L$ for $D$ and

$$
G_{D}(x, y)=\int_{0}^{\infty} p_{D}(t, x, y) d t, \quad x, y \in \mathbb{R}^{d}
$$

is the Green function of $\Delta^{\alpha / 2}$ for $D$.

Let $\mathcal{A}_{d, \gamma}=\Gamma((d-\gamma) / 2) /\left(2^{\gamma} \pi^{d / 2}|\Gamma(\gamma / 2)|\right)$. The Lévy measure of the semigroup generated by $\Delta^{\alpha / 2}$ is given by

$$
v(y)=\mathcal{A}_{d,-\alpha}|y|^{-d-\alpha}, \quad y \in \mathbb{R}^{d} .
$$

The Poisson kernel of $L$ for $D$ may be introduced, like in [10, (38)], by the IkedaWatanabe formula

$$
\tilde{P}_{D}(x, y)=\int_{D} \tilde{G}_{D}(x, z) v(y-z) d z, \quad x \in D, y \in D^{c} .
$$


It is equal to the density of the $L$-harmonic measure for $D$, i.e. if $\tilde{X}_{t}$ is a stochastic process with generator $L$, then

$$
\mathbb{P}^{x}\left(\tilde{X}_{\tilde{\tau}_{D}} \in A\right)=\int_{A} \tilde{P}_{D}(x, y) d y
$$

for any Borel set $A \subset(\bar{D})^{c}$ and $\tilde{\tau}_{D}=\inf \left\{t>0: \tilde{X}_{t} \notin D\right\}$, see [10, (39)]. The Poisson kernel of $\Delta^{\alpha / 2}$ for $D$ is denoted in the paper by $P_{D}(x, y)$ and it may be defined by the formula (2) with $G_{D}$ instead of $\tilde{G}_{D}$. If $B=B(a, r)$ then $P_{B}(x, y)$ is given by

$$
P_{B}(x, y)=C_{d, \alpha}\left[\frac{r^{2}-|x-a|^{2}}{|y-a|^{2}-r^{2}}\right]^{\alpha / 2} \frac{1}{|x-y|^{d}}, \quad x \in B, y \in(\bar{B})^{c} \text {, }
$$

where $C_{d, \alpha}=\Gamma(d / 2) \pi^{-1-d / 2} \sin (\pi \alpha / 2)$.

A Borel function $h$ on $\mathbb{R}^{d}$ is said to be $L$-harmonic on $D$ if for each bounded open set $B$ with $\bar{B} \subset D$ and for $x \in B$ we have

$$
h(x)=\mathbb{E}^{x} h\left(\tilde{X}_{\tilde{\tau}_{B}}\right)
$$

where the last integral is absolutely convergent. If, in addition, $h \equiv 0$ on $D^{c}$ then it is called singular L-harmonic on $D$. On the other hand $h$ is called regular L-harmonic on $D$ if

$$
h(x)=\mathbb{E}^{x} h\left(\tilde{X}_{\tilde{\tau}_{D}}\right)
$$

The harmonic functions of $\Delta^{\alpha / 2}$ on $D$ (called in the paper $\alpha$-harmonic functions) are defined analogously. Their basic properties may be found in the monography [7].

Throughout this paper, like in [10], we suppose $1<\alpha<2$, unless stated otherwise. We consider an open bounded set $D$ of class $\mathcal{C}^{1,1}$ and a vector field $b \in \mathcal{K}_{d}^{\alpha-1}$ on $\mathbb{R}^{d}$, i.e.

$$
\lim _{\epsilon \rightarrow 0} \sup _{x \in \mathbb{R}^{d}} \int_{|x-z|<\epsilon}|b(z)||x-z|^{\alpha-1-d} d z=0 .
$$

We fix throughout this paper a point $x_{0} \in D$ and define the Martin kernel of $\Delta^{\alpha / 2}$ for $D$ by

$$
M_{D}(x, Q)=\lim _{y \rightarrow Q} \frac{G_{D}(x, y)}{G_{D}\left(x_{0}, y\right)}, \quad x \in D, Q \in \partial D .
$$


The $L$-Martin kernel is defined by

$$
\tilde{M}_{D}(x, Q)=\lim _{y \rightarrow Q} \frac{\tilde{G}_{D}(x, y)}{\tilde{G}_{D}\left(x_{0}, y\right)}, \quad x \in D, Q \in \partial D
$$

and we show in Sect. 3 its existence.

The starting point of the research contained in this paper are the following mutual estimates of Green functions and Poisson kernels of $L$ and $\Delta^{\alpha / 2}$ (see [10, Theorem 1 and (72)]).

Comparability Theorem. There exists a constant $C=C(\alpha, b, D)$ such that for all $x, y \in D$ and $z \in(\bar{D})^{c}$,

$$
\begin{gathered}
C^{-1} G_{D}(x, y) \leq \tilde{G}_{D}(x, y) \leq C G_{D}(x, y), \\
C^{-1} P_{D}(x, z) \leq \tilde{P}_{D}(x, z) \leq C P_{D}(x, z) .
\end{gathered}
$$

One of the main elements of the proof of (4) is the following perturbation formula, that will be also very useful in our present work (see [10, Lemma 12]).

Perturbation formula for Green functions. Let $x, y \in \mathbb{R}^{d}, x \neq y$. We have

$$
\tilde{G}_{D}(x, y)=G_{D}(x, y)+\int_{D} \tilde{G}_{D}(x, z) b(z) \cdot \nabla_{z} G_{D}(z, y) d z
$$

\subsection{Outline of the paper}

We start our paper by proving in Sect. 2.1 a generalization of the Comparability Theorem: according to Lemma 1, the constant $C$ in the estimates (4) may be chosen the same for sets $D_{r}$ sufficiently close to $D$. The same phenomenon holds also for the Poisson kernels $\tilde{P}_{D}(x, y)$ and $P_{D}(x, y)$. In Section 2.2 we prove a uniform integrability result, that will be needed in proving the main results of the paper, contained in Sections 3 and 4.

In Section 3 we develop the Martin theory of $L$-harmonic functions. We prove the existence of the $L$-Martin kernel which is $L$-harmonic (Theorems 8 and 12). Next we obtain the Martin representation of singular $L$-harmonic non-negative functions on $D$, see Theorem 13.

The formula (6) allows us to prove very useful perturbation formulas for Martin kernels (15), Poisson kernels (18) and singular $\alpha$-harmonic functions (29). In Section $3.4,(6)$ and (15) are proved in the diffusion case $\alpha=2$. Also a perturbation formula (34) for the $L$-Poisson kernel is derived.

Section 4 is devoted to an important fine boundary property of singular $L$-harmonic functions: the Relative Fatou Theorem (Theorem 23). We provide a proof of this theorem based on the perturbation formula for singular $L$-harmonic functions (29). 


\section{Preparatory results}

In this section we prove some results, interesting independently, that will be useful in proving the main results of the paper, coming in the next sections. In what follows $D$ is a bounded $\mathcal{C}^{1,1}$ open set.

2.1 Uniform comparability of Green functions and Poisson kernels

For $r \geq 0$ define

$$
D_{r}=\left\{x \in D: \delta_{D}(x)>r\right\}
$$

When $r$ is sufficiently small, then $D_{r}$ is also a $\mathcal{C}^{1,1}$ open set, see [40, Lemma 5], and one may show that the localization radius of $D_{r}$ varies continuously with respect to $r$.

In the sequel we will often use the estimates of the Green function $([20,36]$, see also [28]) of a $\mathcal{C}^{1,1}$ open set

$$
G_{D}(y, z) \approx|y-z|^{\alpha-d}\left(\frac{\delta_{D}(y)^{\alpha / 2} \delta_{D}(z)^{\alpha / 2}}{|y-z|^{\alpha}} \wedge 1\right),
$$

and of the Martin kernel ([21])

$$
M_{D}(x, Q) \approx \frac{\delta_{D}(x)^{\alpha / 2}}{|x-Q|^{d}}
$$

Moreover, in the stable case, the estimates (7) are uniform when we consider the sets $D_{r}$ sufficiently close to $D$, i.e. there exist constants $c, \epsilon_{0}>0$ depending only on $D$ and $\alpha$ such that for all $r \in\left[0, \epsilon_{0}\right]$ and $x, y \in D_{r}$ we have

$$
\begin{aligned}
& c^{-1}|y-z|^{\alpha-d}\left(\frac{\delta_{D_{r}}(y)^{\alpha / 2} \delta_{D_{r}}(z)^{\alpha / 2}}{|y-z|^{\alpha}} \wedge 1\right) \\
& \quad \leq G_{D_{r}}(y, z) \leq c|y-z|^{\alpha-d}\left(\frac{\delta_{D_{r}}(y)^{\alpha / 2} \delta_{D_{r}}(z)^{\alpha / 2}}{|y-z|^{\alpha}} \wedge 1\right),
\end{aligned}
$$

see [28, Theorem 21] and [40, Lemma 5]. We will now show analogous uniformity of constants for the fractional Laplacian with a gradient perturbation.

Lemma 1 (i) There exist constants $c, \epsilon_{0}>0$ depending only on $D$ and $\alpha$ such that for all $r \in\left[0, \epsilon_{0}\right]$ and $x, y \in D_{r}$ we have

$$
c^{-1} G_{D_{r}}(x, y) \leq \tilde{G}_{D_{r}}(x, y) \leq c G_{D_{r}}(x, y)
$$


(ii) There exist constants $C, \epsilon_{0}>0$ depending only on $D$ and $\alpha$ such that for all $r \in\left[0, \epsilon_{0}\right], x \in D_{r}$ and $y \in D_{r}^{c}$ we have

$$
C^{-1} P_{D_{r}}(x, y) \leq \tilde{P}_{D_{r}}(x, y) \leq C P_{D_{r}}(x, y)
$$

Proof In order to show (i) we follow the proof of the Theorem 1 in [10]. We analyse below its crucial points.

1. Comparison of Green functions $\tilde{G}_{S}(x, y)$ and $G_{S}(x, y)$ for "small" sets $S$, [10, Lemma 13], based on estimates from [10, Lemma 11]. Thanks to property (9), we see that the comparison of Green functions $\tilde{G}_{S_{r}}(x, y)$ and $G_{S_{r}}(x, y)$ for small sets $S$ holds with a common constant $c$, when $r \in\left[0, \epsilon_{0}\right]$.

2. Harnack inequalities for L and the Boundary Harnack Principle, [10, Lemmas $15,16]$. Thanks to 1 ., we get them uniformly with respect to $r \in\left[0, \epsilon_{0}\right]$.

3. Now the proof of (i) for any $\mathcal{C}^{1,1}$ open set $D$ is the same as in Section 5 of [10].

The part (ii) is implied by (i), applying the Ikeda-Watanabe formula for the Poisson kernel $\tilde{P}_{D}$, see [10, Lemma 6 and (39)]. Recall that the Lévy system for the process $\tilde{X}_{t}$ is given by the Lévy measure of the $\alpha$-stable process $X_{t}$.

An immediate consequence of Lemma 1 and [28, Theorem 22] is the following uniform estimate of the Poisson kernels of $L$ for $D_{r}$.

Corollary 2 There exist positive constants $C, \epsilon_{0}$ depending only on $D, \alpha$ and $b$ such that for all $r \in\left[0, \epsilon_{0}\right], x \in D_{r}$ and $y \in D_{r}^{c}$, we have

$$
\frac{C^{-1} \delta_{D_{r}}^{\alpha / 2}(x)}{\delta_{D_{r}}^{\alpha / 2}(y)\left(1+\delta_{D_{r}}(y)\right)^{\alpha / 2}|x-y|^{d}} \leq \tilde{P}_{D_{r}}(x, y) \leq \frac{C \delta_{D_{r}}^{\alpha / 2}(x)}{\delta_{D_{r}}^{\alpha / 2}(y)\left(1+\delta_{D_{r}}(y)\right)^{\alpha / 2}|x-y|^{d}} .
$$

\subsection{Derivatives of the Poisson kernel of $\Delta^{\alpha / 2}$}

In this section we prove useful gradient estimates for the Poisson kernel of $\Delta^{\alpha / 2}$ for $D, 0<\alpha<2$. Consider a ball $B=B\left(\xi_{0}, r\right) \subset \bar{B} \subset D$ and let $P_{B}$ be the Poisson kernel of $\Delta^{\alpha / 2}$ for $B$. By [12, Lemma 3.1],

$$
\left|\nabla_{x} P_{B}(x, y)\right| \leq(d+\alpha) \frac{P_{B}(x, y)}{r-\left|x-\xi_{0}\right|}, \quad x \in B, y \in(\bar{B})^{c}
$$

We will now show analogous estimate for $\mathcal{C}^{1,1}$ bounded open sets.

Lemma 3 Suppose $0<\alpha<2$. Let $D$ be a bounded $\mathcal{C}^{1,1}$ open set in $\mathbb{R}^{d}$ and let $P_{D}(x, y)$ be the Poisson kernel of $\Delta^{\alpha / 2}$ for $D$. Then we have

$$
\left|\nabla_{x} P_{D}(x, y)\right| \leq(\alpha+d) \frac{P_{D}(x, y)}{\delta_{D}(x)}, \quad x \in D, y \in(\bar{D})^{c} .
$$


Proof For $x \in D$ denote $B_{x}=B\left(x, \delta_{D}(x)\right)$. In view of [11, (29)] we have

$$
P_{D}(x, y)=P_{B_{x}}(x, y)+\int_{B_{x}^{c}} P_{B_{x}}(x, z) P_{D}(z, y) d z .
$$

By (10) and bounded convergence we have

$$
\begin{aligned}
& \left|\nabla_{x} P_{D}(x, y)\right| \leq\left|\nabla_{x} P_{B_{x}}(x, y)\right|+\left|\nabla_{x} \int_{B_{x}^{c}} P_{B_{x}}(x, z) P_{D}(z, y) d z\right| \\
& \quad \leq(d+\alpha) \frac{P_{B_{x}}(x, y)}{\delta_{D}(x)}+\int_{B_{x}^{c}}\left|\nabla_{x} P_{B_{x}}(x, z)\right| P_{D}(z, y) d z \leq(d+\alpha) \frac{P_{D}(x, y)}{\delta_{D}(x)} .
\end{aligned}
$$

From (10) and the dominated convergence theorem it follows, that if $f$ is $\alpha$-harmonic in $D$ then

$$
\frac{\partial}{\partial x_{i}} f(x)=\int_{B^{c}} \frac{\partial}{\partial x_{i}} P_{B}(x, y) f(y) d y, \quad i=1, \ldots, d .
$$

The estimates (10) and (12) give the following result ([12, Lemma 3.2]).

Lemma 4 Let $U$ be an arbitrary open set in $\mathbb{R}^{d}$ and let $\alpha \in(0,2)$. For every nonnegative function $u$ on $\mathbb{R}^{d}$ which is $\alpha$-harmonic in $U$, we have

$$
|\nabla u(x)| \leq d \frac{u(x)}{\delta_{U}(x)}, \quad x \in U
$$

Since $G_{U}(\cdot, y)$ is $\alpha$-harmonic in $U \backslash\{y\}$, for every $y \in U$ we obtain

$$
\left|\nabla_{x} G_{U}(x, y)\right| \leq d \frac{G_{U}(x, y)}{\delta_{U}(x) \wedge|x-y|}, \quad x, y \in U, \quad x \neq y .
$$

\subsection{A uniform integrability result}

Recall [10, Lemma 9 ].

Lemma $5 G_{D}(y, w) /[\delta(w) \wedge|y-w|]$ is uniformly in y integrable against $|b(w)| d w$. In the next lemma we will show a similar property for the family of functions $G_{D_{2^{-n}}}(x, w) M_{D}(w, Q) \delta_{D_{2^{-n}}}(w)^{-1}$.

Lemma 6 Let $x \in D$ be fixed. There exists $N=N(D, x) \in \mathbb{N}$ such that the functions

$$
G_{D_{2-n}}(x, w) M_{D}(w, Q) \delta_{D_{2-n}}(w)^{-1}
$$

are uniformly in $Q \in \partial D$ and $n>N$ integrable against $|b(w)| d w$. 
Proof In view of the $\mathcal{C}^{1,1}$ property of $D$ and of the estimates (8) and (9) of $M_{D}(w, Q)$ and $G_{D_{2-n}}(x, w)$, we can choose $N=N(D, x) \in \mathbb{N}$ sufficiently large, such that for all $n>N$, we have

$$
\begin{aligned}
\frac{G_{D_{2^{-n}}}(x, w) M_{D}(w, Q)}{\delta_{D_{2^{-n}}}(w)} & \leq \frac{c \mathbf{1}_{D_{2^{-n}}}(w) \delta_{D}(w)^{\alpha / 2}}{\delta_{D_{2^{-n}}}(w)^{1-\alpha / 2}|w-Q|^{d}|w-x|^{d-\alpha}} \\
& \leq \tilde{c} \mathbf{1}_{D_{2^{-n}}}(w)\left(|w-x|^{\alpha-d}+\frac{\delta_{D}(w)^{\alpha / 2}}{\delta_{D_{2^{-n}}}(w)^{1-\alpha / 2}|w-Q|^{d}}\right),
\end{aligned}
$$

where $c$ and $\tilde{c}$ depends only on $D, \alpha$ and $x$. The first term in the parentheses is integrable against $|b(w)| d w$ independently of $Q, n$, so we only need to consider the second one. For $w \in D_{2^{-N}}$ and $Q \in \partial D$, we have

$$
\frac{\delta_{D}(w)^{\alpha / 2}}{\delta_{D_{2-n}}(w)^{1-\alpha / 2}|w-Q|^{d}} \leq \operatorname{diam}(D)^{\alpha / 2} 2^{(N+1)(d+1-\alpha / 2)},
$$

and for $w \in D_{2^{-n}} \backslash D_{2^{-N}}, Q \in \partial D$, we get

$$
\begin{aligned}
\frac{\delta_{D}(w)^{\alpha / 2}}{\delta_{D_{2}-n}(w)^{1-\alpha / 2}|w-Q|^{d}} & =\frac{\left(\delta_{D_{2}-n}(w)+2^{-n}\right)^{\alpha / 2}}{\delta_{D_{2^{-n}}}(w)^{1-\alpha / 2}|w-Q|^{d}} \\
& \leq 2^{\alpha / 2}\left(|w-Q|^{\alpha-d-1}+\frac{2^{-n \alpha / 2}}{\delta_{D_{2-n}}(w)^{1-\alpha / 2}|w-Q|^{d}}\right) .
\end{aligned}
$$

Since $\mathbf{1}_{D_{2}-n}(w)|w-Q|^{\alpha-d-1}$ is uniformly in $Q, n$ integrable against $|b(w)| d w$, we can restrict our attention to the function

$$
H_{n}(w, Q)=\frac{2^{-n \alpha / 2} \mathbf{1}_{D_{2-n}}(w)}{\delta_{D_{2-n}}(w)^{1-\alpha / 2}|w-Q|^{d}} .
$$

Let $R>N, R \in \mathbb{N}$. For $k, m, n \in \mathbb{N}, k \geq R, m \geq N, n>N$, we define

$$
\begin{gathered}
W_{k, m}^{n}(Q, R)=\left\{w \in D_{2^{-n}}: \frac{1}{2^{k+1}}<\delta_{D_{2^{-n}}}(w) \leq \frac{1}{2^{k}}, \quad \frac{1}{2^{m+1}}<|w-Q| \leq \frac{1}{2^{m}}\right\}, \\
W_{k}^{n}(Q, R)=\left\{w \in D_{2^{-n}}: \frac{1}{2^{k+1}}<\delta_{D_{2^{-n}}}(w) \leq \frac{1}{2^{k}}, \quad|w-Q|>\frac{1}{2^{N}}\right\} .
\end{gathered}
$$

We note that $W_{k, m}^{n}(Q, R)=\emptyset$ for $k<m$ or $m \geq n$. $W_{k, m}^{n}(Q, R)$ can be covered by $c_{1}\left(2^{k-m}\right)^{d-1}$ balls of radii $2^{-k}$, where $c_{1}=c_{1}(D)$. For $r>0$, denote

$$
K_{r}=\sup _{z \in \mathbb{R}^{d}} \int_{B(z, r)}|b(w)||z-w|^{\alpha-d-1} d w .
$$


Then, $K_{r} \rightarrow 0$ as $r \downarrow 0$. We have

$$
\begin{aligned}
& \int_{W_{k, m}^{n}(Q, R)} H_{n}(w, Q)|b(w)| d w \\
& \leq\left(2^{k+1}\right)^{1-\alpha / 2}\left(2^{m+1}\right)^{d} 2^{-n \alpha / 2} c_{1}\left(2^{k-m}\right)^{d-1} \sup _{z \in D} \int_{B\left(z, 2^{-k}\right)}|b(w)| d w \\
& \leq\left(2^{k+1}\right)^{1-\alpha / 2}\left(2^{m+1}\right)^{d} 2^{-n \alpha / 2} c_{1}\left(2^{k-m}\right)^{d-1}\left(2^{k}\right)^{\alpha-d-1} K_{2^{-k}} \\
& \leq c_{2} K_{2^{-R}}\left(2^{k}\right)^{\alpha / 2-1} 2^{m} 2^{-n \alpha / 2},
\end{aligned}
$$

where $c_{2}=c_{2}(D, b, \alpha)$. Furthermore, $W_{k}^{n}(Q, R)$ can be covered by $c_{3}\left(2^{k}\right)^{d-1}$ balls of radii $2^{-k}$, where $c_{3}=c_{3}(D)$, and thus

$$
\begin{aligned}
& \int_{W_{k}^{n}(Q, R)} H_{n}(w, Q)|b(w)| d w \\
& \quad \leq\left(2^{k+1}\right)^{1-\alpha / 2} 2^{N d} 2^{-n \alpha / 2} c_{3}\left(2^{k}\right)^{d-1} \sup _{z \in D} \int_{B\left(z, 2^{-k}\right)}|b(w)| d w \\
& \quad \leq\left(2^{k+1}\right)^{1-\alpha / 2} 2^{N d} 2^{-n \alpha / 2} c_{3}\left(2^{k}\right)^{d-1}\left(2^{k}\right)^{\alpha-d-1} K_{2^{-k}} \\
& \quad \leq c_{4} K_{2^{-R}}\left(2^{k}\right)^{\alpha / 2-1} 2^{-n \alpha / 2},
\end{aligned}
$$

where $c_{4}=c_{4}(D, b, \alpha)$. Let $A_{R}^{n}=\left\{w \in D_{2^{-n}}: \delta_{D_{2^{-n}}}(w) \leq 2^{-R}\right\}$. Then,

$$
A_{R}^{n}=\sum_{k=R}^{\infty} W_{k}^{n}(Q, R)+\sum_{m=N}^{n-1} \sum_{k=R \vee m}^{\infty} W_{k, m}^{n}(Q, R),
$$

and we obtain

$$
\begin{aligned}
& \int_{A_{R}^{n}} H_{n}(w, Q)|b(w)| d w \\
& \leq c_{4} K_{2^{-R}} 2^{-n \alpha / 2} \sum_{k=R}^{\infty}\left(2^{k}\right)^{\alpha / 2-1}+\sum_{m=N}^{n-1} \sum_{k=R \vee m}^{\infty} c_{2} K_{2^{-R}}\left(2^{k}\right)^{\alpha / 2-1} 2^{m} 2^{-n \alpha / 2} \\
& \leq c_{5} K_{2^{-R}}\left(2^{-n \alpha / 2}\left(2^{R}\right)^{\alpha / 2-1}+\sum_{m=N}^{n-1}\left(2^{n-m}\right)^{-\alpha / 2}\right) \leq c_{6} K_{2^{-R}},
\end{aligned}
$$

where $c_{6}=c_{6}(D, b, \alpha)$. For $w \in D_{2^{-n}} \backslash A_{R}^{n}$, we have 


$$
H_{n}(w, Q)<2^{-n \alpha / 2}\left(2^{R}\right)^{1-\alpha / 2}\left(\frac{1}{2^{R}}+\frac{1}{2^{n}}\right)^{-d}<4^{d R}
$$

so $B_{R}^{n}(Q):=\left\{w: H_{n}(w, Q)>4^{d R}\right\} \subset A_{R}^{n}$ for all $Q \in \partial D$ and $n>N$. Therefore,

$$
\lim _{R \rightarrow \infty} \sup _{Q \in \partial D, n>N} \int_{B_{R}^{n}(Q)} H_{n}(w, Q)|b(w)| d w \leq \lim _{R \rightarrow \infty} c_{6} K_{2^{-R}}=0 .
$$

\section{Martin kernel and Martin representation}

In this section we will discuss first the existence and the properties of the Martin kernel of $L$ for a $\mathcal{C}^{1,1}$ bounded open set $D$. Next we will investigate the Martin representation for non-negative singular $L$-harmonic functions on $D$.

\subsection{Existence and Perturbation formula for the $L$-Martin kernel}

In order to prove the existence of the $L$-Martin kernel, we will need the following property of the Green function for $\Delta^{\alpha / 2}$.

Lemma 7 For all $x \in D$ and $Q \in \partial D$ we have

$$
\lim _{y \rightarrow Q} \frac{\nabla_{x} G_{D}(x, y)}{G_{D}\left(x_{0}, y\right)}=\nabla_{x} M_{D}(x, Q)
$$

Proof Let $z \in D, Q \in \partial D$ and choose $r>0$ such that $\overline{B(z, r)} \subset D$ and $B(z, r) \cap$ $B(Q, r)=\emptyset$. Since $G_{D}(\cdot, y)$ is $\alpha$-harmonic in $B(z, r)$ for $y \in B(Q, r) \cap D$, by (12), we have

$$
\begin{aligned}
\frac{\nabla_{x} G_{D}(x, y)}{G_{D}\left(x_{0}, y\right)} & =\nabla_{x} \int_{B(z, r)^{c}} P_{B(z, r)}(x, w) \frac{G_{D}(w, y)}{G_{D}\left(x_{0}, y\right)} d w \\
& =\int_{B(z, r)^{c}} \nabla_{x} P_{B(z, r)}(x, w) \frac{G_{D}(w, y)}{G_{D}\left(x_{0}, y\right)} d w, \quad x \in B(z, r) .
\end{aligned}
$$

Furthermore, by (10) and (7),

$$
\left|\nabla_{x} P_{B(z, r)}(x, w)\right| \frac{G_{D}(w, y)}{G_{D}\left(x_{0}, y\right)} \leq C \frac{P_{B(z, r)}(x, w)}{r-|x-z|} \frac{G_{D}(w, y)}{\delta_{D}(y)^{\alpha / 2}} .
$$


We now use the estimate $[10,(25)]$ and by considering the cases $\delta_{D}(w)>|w-y|$ and $\delta_{D}(w) \leq|w-y|$ we get $\frac{G_{D}(w, y)}{\delta_{D}(y)^{\alpha / 2}} \leq C|w-y|^{\alpha / 2-d}$. Hence the last term is uniformly in $y \in B(Q, r / 2) \cap D$ integrable against $d w$, and thus

$$
\begin{aligned}
& \lim _{y \rightarrow Q} \int_{B(z, r)^{c}} \nabla_{x} P_{B(z, r)}(x, w) \frac{G_{D}(w, y)}{G_{D}\left(x_{0}, y\right)} d w \\
& =\int_{B(z, r)^{c}} \nabla_{x} P_{B(z, r)}(x, w) M_{D}(w, Q) d w=\nabla_{x} M_{D}(x, Q) .
\end{aligned}
$$

The last equality follows from (12) and the $\alpha$-harmonicity of the Martin kernel.

Thanks to Lemma 7, we obtain the main result of this subsection.

Theorem 8 Let $x \in D$ and $Q \in \partial D$. Let $M_{D}(x, Q)$ be the Martin kernel of $\Delta^{\alpha / 2}$ for D. Denote

$$
l_{D}(x, Q)=M_{D}(x, Q)+\int_{D} \tilde{G}_{D}(x, z) b(z) \cdot \nabla_{z} M_{D}(z, Q) d z
$$

The function $l_{D}(x, Q)$ is well defined for $x \in D$ and $Q \in \partial D$ and $l_{D}(x, Q)>0$. Moreover the following limit exists and equals:

$$
\lim _{y \rightarrow Q} \frac{\tilde{G}_{D}(x, y)}{\tilde{G}_{D}\left(x_{0}, y\right)}=\frac{l_{D}(x, Q)}{l_{D}\left(x_{0}, Q\right)} .
$$

Thus the Martin kernel of $L=\Delta^{\alpha / 2}+b \cdot \nabla$ for $D$ exists and equals

$$
\tilde{M}_{D}(x, Q)=\frac{1}{l_{D}\left(x_{0}, Q\right)}\left[M_{D}(x, Q)+\int_{D} \tilde{G}_{D}(x, z) b(z) \cdot \nabla_{z} M_{D}(z, Q) d z\right] .
$$

Proof We divide the perturbation formula (6) for the Green function $\tilde{G}_{D}(x, y)$ by $G_{D}\left(x_{0}, y\right)$ and let $y \rightarrow Q$.

The exchange of $\lim _{y \rightarrow Q}$ and $\int_{D}$ is justified by Lemma 11 of [10], see the formula (49) in its proof. Note that by the Boundary Harnack Principle, $G_{D}\left(x_{0}, y\right) \approx G_{D}(x, y)$ when $y \in B\left(Q, \epsilon_{0}\right)$, a sufficiently small ball around $Q$. We also use the estimates (7), (14) and (4).

The exchange of $\lim _{y \rightarrow Q}$ and $\nabla_{z}$ is justified by Lemma 7. Finally,

$$
\lim _{y \rightarrow Q} \frac{\tilde{G}_{D}(x, y)}{G_{D}\left(x_{0}, y\right)}=M_{D}(x, Q)+\int_{D} \tilde{G}_{D}(x, z) b(z) \cdot \nabla M_{D}(z, Q)=l_{D}(x, Q) .
$$


The strict positivity of the function $l_{D}(x, Q)$ follows from (4), which implies that there exists $a>0$ such that

$$
l_{D}(x, Q) \geq a M_{D}(x, Q)>0 .
$$

Now, we consider the quotient

$$
\frac{\tilde{G}_{D}(x, y)}{\tilde{G}_{D}\left(x_{0}, y\right)}=\frac{\tilde{G}_{D}(x, y)}{G_{D}\left(x_{0}, y\right)} \frac{G_{D}\left(x_{0}, y\right)}{\tilde{G}_{D}\left(x_{0}, y\right)} \rightarrow \frac{l_{D}(x, Q)}{l_{D}\left(x_{0}, Q\right)},
$$

when $y \rightarrow Q$.

Directly from the definition of $\tilde{M}_{D}(x, Q)$ and (4) we obtain the following corollary.

Corollary 9 There is a constant $c$ such that for all $x \in D$ and $Q \in \partial D$,

$$
c^{-1} M_{D}(x, Q) \leq \tilde{M}_{D}(x, Q) \leq c M_{D}(x, Q) .
$$

\subsection{Properties of the $L$-Martin kernel}

We will now study further properties of the Martin kernel of $L$ for $D$. We start with the following useful formulas.

Lemma 10 Consider a $\mathcal{C}^{1,1}$ open set $U \subset \bar{U} \subset D$.

(i) (Perturbation formula for the Poisson kernel) For all $x \in U, z \in(\bar{U})^{c}$

$$
\tilde{P}_{U}(x, z)=P_{U}(x, z)+\int_{U} \tilde{G}_{U}(x, w) b(w) \cdot \nabla_{w} P_{U}(w, z) d w
$$

(ii) Let $Q \in \partial D$. We have the following expression for the L-Poisson integral of the Martin kernel of $\Delta^{\alpha / 2}$ :

$$
\begin{aligned}
\tilde{P}_{U} M_{D}(x, Q) & :=\int_{U^{c}} \tilde{P}_{U}(x, y) M_{D}(y, Q) d y \\
& =M_{D}(x, Q)+\int_{U} \tilde{G}_{U}(x, z) b(z) \cdot \nabla_{z} M_{D}(z, Q) d z, \quad x \in U .
\end{aligned}
$$


Proof In the following we apply the Ikeda-Watanabe formula for the Poisson kernels $\tilde{P}_{U}$ and $P_{U}$. By (6) and Fubini's theorem, for any $x \in U$ and $z \in U^{c}$,

$$
\begin{aligned}
\tilde{P}_{U}(x, z) & =\int_{U} \mathcal{A}_{d, \alpha} \frac{\tilde{G}_{U}(x, y)}{|z-y|^{d+\alpha}} d y \\
& =\int_{U} \frac{\mathcal{A}_{d, \alpha}}{|z-y|^{d+\alpha}}\left[G_{U}(x, y)+\int_{U} \tilde{G}_{U}(x, w) b(w) \cdot \nabla_{w} G_{U}(w, y) d w\right] d y \\
& =P_{U}(x, z)+\int_{U} \tilde{G}_{U}(x, w) b(w) \cdot \nabla_{w} P_{U}(w, z) d w .
\end{aligned}
$$

For the necessary exchanges of order of integration and derivation in the last formula, we apply (14), (4), Lemma 5 and bounded convergence theorem. In order to prove (ii), we use (i) and insert the formula (18) in $\int_{U^{c}} \tilde{P}_{U}(x, y) M_{D}(y, Q) d y$. We obtain

$$
\int_{U^{c}} \tilde{P}_{U}(x, y) M_{D}(y, Q) d y=M_{D}(x, Q)+\int_{U} \tilde{G}_{U}(x, z) b(z) \cdot \nabla_{z} M_{D}(z, Q) d z .
$$

In the last equality the use of Fubini theorem and the exchange of $\int$ and $\nabla$ are justified by (11), (8), Lemma 5 and bounded convergence.

Lemma 11 The Martin kernel $\tilde{M}_{D}(\cdot, \cdot)$ is jointly continuous on $D \times \partial D$.

Proof By Theorem 8 and the continuity of $M_{D}(\cdot, \cdot)$, it suffices to show the joint continuity on $D \times \partial D$ of the function

$$
f(x, Q)=\int_{D} \tilde{G}_{D}(x, z) b(z) \cdot \nabla_{z} M_{D}(z, Q) d z
$$

Let $z \in D$. By (12) and the $\alpha$-harmonicity of $M_{D}(\cdot, Q)$, for $r>0$ sufficiently small, we have

$$
\begin{aligned}
\nabla_{z} M_{D}(z, Q) & =\nabla_{z} \int_{B(z, r)^{c}} P_{B(z, r)}(z, w) M_{D}(w, Q) d w \\
& =\int_{B(z, r)^{c}} \nabla_{z} P_{B(z, r)}(z, w) M_{D}(w, Q) d w .
\end{aligned}
$$

From (10) and (8) it follows, that $\nabla_{z} P_{B(z, r)}(z, w) M_{D}(w, Q)$ is uniformly in $Q$ integrable against $d w$. This implies that $\nabla_{z} M_{D}(z, \cdot)$ is continuous on $\partial D$ for every $z \in D$. Let now $x \in D$ and choose $r>0$ such that $\overline{B(x, r)} \subset D$. By (4), (7), (13) and (8), for all $y \in B(x, r), z \in D$ and $Q \in \partial D$, we have 


$$
\tilde{G}_{D}(y, z)\left|\nabla_{z} M_{D}(z, Q)\right| \leq \frac{C \delta_{D}(z)^{\alpha-1}}{|y-z|^{d-\alpha}|z-Q|^{d}} \leq \frac{C}{|y-z|^{d-\alpha}|z-Q|^{d+1-\alpha}} .
$$

Hence, $\tilde{G}_{D}(y, z)\left|\nabla_{z} M_{D}(z, Q)\right|$ is uniformly in $y \in B(x, r)$ and $Q \in \partial D$ integrable against $|b(z)| d z$, which gives the continuity of $f(\cdot, \cdot)$.

We will now use Lemma 10 to show $L$-harmonicity of $\tilde{M}_{D}(\cdot, Q)$.

Theorem 12 For every $Q \in \partial D$ the Martin kernel $\tilde{M}_{D}(x, Q)$ is a singular $L$-harmonic function of $x$ on $D$.

Proof First consider a $\mathcal{C}^{1,1}$ open set $U=D_{r}$. We note that by the strong Markov property, see e.g. [10, p. 466],

$$
\tilde{G}_{D}(x, w)=\tilde{G}_{U}(x, w)+\int_{U^{c}} \tilde{P}_{U}(x, z) \tilde{G}_{D}(z, w) d z .
$$

By (19), (18), (21) and Fubini’s theorem

$$
\begin{aligned}
\tilde{P}_{U} l_{D}(x, Q)= & \int_{U^{c}} \tilde{P}_{U}(x, z) l_{D}(z, Q) d z \\
= & \int_{U^{c}} \tilde{P}_{U}(x, z) M_{D}(z, Q) d z \\
& +\int_{U^{c}} \tilde{P}_{U}(x, z) \int_{D} \tilde{G}_{D}(z, w) b(w) \cdot \nabla_{w} M_{D}(w, Q) d w d z \\
= & M_{D}(x, Q)+\int_{U^{c}} \int_{U} \tilde{G}_{U}(x, w) b(w) \cdot \nabla_{w} P_{U}(w, z) d w M_{D}(z, Q) d z \\
& +\int_{D}\left[\int_{U^{c}} \tilde{P}_{U}(x, z) \tilde{G}_{D}(z, w) d z\right] b(w) \cdot \nabla_{w} M_{D}(w, Q) d w \\
= & M_{D}(x, Q)+\int_{U} \tilde{G}_{U}(x, w) b(w) \cdot \nabla_{w} M_{D}(w, Q) d w \\
& +\int_{D}\left[\tilde{G}_{D}(x, w)-\tilde{G}_{U}(x, w)\right] b(w) \cdot \nabla_{w} M_{D}(w, Q) d w \\
= & M_{D}(x, Q)+\int_{D} \tilde{G}_{D}(x, w) b(w) \cdot \nabla_{w} M_{D}(w, Q) d w=l_{D}(x, Q) .
\end{aligned}
$$

Thus the function $l_{D}(x, Q)$ is regular $L$-harmonic on each set $U=D_{r}$ for $r$ sufficiently small. By the strong Markov property, it has the mean value property on each open set $U \subset \bar{U} \subset D$. 
Remark 1 Other classical results can be proved for the $L$-Martin kernel $\tilde{M}_{D}(x, Q)$. Let us mention the limit property

$$
\tilde{M}_{D}(x, Q)=\lim _{(\bar{D})^{c} \ni y \rightarrow Q} \frac{\tilde{P}_{D}(x, Q)}{\tilde{P}_{D}\left(x_{0}, Q\right)}
$$

proved for $M_{D}(x, Q)$ in [6, Lemma 7]. The proof goes along the ideas of [6].

\subsection{L-Martin representation}

The objective of this section is to prove the following Martin representation theorem for non-negative singular $L$-harmonic functions on $D$.

Theorem 13 For every non-negative finite measure $v$ on $\partial D$ the function $u$ given by

$$
u(x)=\int_{\partial D} \tilde{M}_{D}(x, Q) d v(Q),
$$

is singular L-harmonic on D. Conversely, if $u$ is non-negative singular L-harmonic on $D$, then there exists a unique non-negative finite measure $v$ on $\partial D$ verifying (22).

Proof The $L$-harmonicity of the Martin integral (22) and the uniqueness of the representation follow from Theorem 12, Lemma 11, (8), (17) and Fubini theorem, in the same way as in the case of the Martin representation for $\alpha$-harmonic functions in [6, proof of Theorem 1]. We will now focus on the existence part. By $L$-harmonicity of $u$ and by (18), we have for each $n$

$$
\begin{aligned}
u(x) & =\int_{D_{1 / n}^{c}} \tilde{P}_{D_{1 / n}}(x, y) u(y) d y \\
& =\int_{D_{1 / n}^{c}} u(y)\left[P_{D_{1 / n}}(x, y)+\int_{D_{1 / n}} \tilde{G}_{D_{1 / n}}(x, w) b(w) \cdot \nabla_{w} P_{D_{1 / n}}(w, y) d w\right] d y .
\end{aligned}
$$

Denote

$$
u_{n}^{*}(x)=\int_{D_{1 / n}^{c}} P_{D_{1 / n}}(x, y) u(y) d y .
$$


By (11), [10, (72)] and Lemma 5, we have

$$
\begin{aligned}
& \int_{D_{1 / n}^{c}} \int_{D_{1 / n}} \tilde{G}_{D_{1 / n}}(x, w)|b(w)|\left|\nabla_{w} P_{D_{1 / n}}(w, y)\right| u(y) d w d y \\
& \quad \leq C \int_{D_{1 / n}} \tilde{G}_{D_{1 / n}}(x, w)|b(w)| \frac{u(w)}{\delta_{D_{1 / n}}(w)} d w<\infty,
\end{aligned}
$$

where $C=C\left(\alpha, b, D_{1 / n}\right)>0$. Hence, by Fubini theorem

$$
u(x)=u_{n}^{*}(x)+\int_{D_{1 / n}} \tilde{G}_{D_{1 / n}}(x, w) b(w) \cdot \int_{D_{1 / n}^{c}} \nabla_{w} P_{D_{1 / n}}(w, y) u(y) d y d w
$$

The function $u_{n}^{*}$ is $\alpha$-harmonic on $D_{1 / n}$, so it is differentiable. In order to justify the exchange of $\int$ and $\nabla$ in the last integral we fix $w \in D_{1 / n}$. Then, by (11) and (5), for $\varepsilon>0$ sufficiently small and all $w^{\prime} \in B(w, \varepsilon)$ and $y \in D_{1 / n}^{c}$, we have

$$
\left|\nabla_{w^{\prime}} P_{D_{1 / n}}\left(w^{\prime}, y\right) u(y)\right| \leq C \frac{u(y)}{\delta_{D_{1 / n}}(y)^{\alpha / 2}},
$$

where $C=C\left(\alpha, b, D_{1 / n}, \varepsilon\right)>0$. Since the last term is integrable on $D_{1 / n}^{c}$, by the dominated convergence, we obtain

$$
u(x)=u_{n}^{*}(x)+\int_{D} \tilde{G}_{D_{1 / n}}(x, w) b(w) \cdot \nabla u_{n}^{*}(w) d w .
$$

We now study the sequence $u_{n}^{*}(x)$ in the same way as K. Bogdan [6] in the proof of the existence part of the $\Delta^{\alpha / 2}-$ Martin representation, with the difference that in our case the function $u$ under the integral defining $u_{n}^{*}$ is not $\alpha$-harmonic.

By Ikeda-Watanabe formula (2), we have

$$
u_{n}^{*}(x)=\int_{D_{1 / n}^{c}} P_{D_{1 / n}}(x, y) u(y) d y=\int_{D_{1 / n}^{c}} \int_{D_{1 / n}} u(y) \mathcal{A}_{d, \alpha} \frac{G_{D_{1 / n}}(x, \xi)}{|\xi-y|^{d+\alpha}} d \xi d y
$$

Set $\mu_{n}(d \xi)=\mathcal{A}_{d, \alpha} G_{D_{1 / n}}\left(x_{0}, \xi\right) \int_{D_{1 / n}} \frac{u(y)}{|\xi-y|^{d+\alpha}} d y d \xi$. Lemma 1 implies that

$$
\mu_{n}\left(\mathbb{R}^{d}\right)=\int_{D_{1 / n}^{c}} P_{D_{1 / n}}\left(x_{0}, y\right) u(y) d y \leq C \int_{D_{1 / n}^{c}} \tilde{P}_{D_{1 / n}}\left(x_{0}, y\right) u(y) d y=c u\left(x_{0}\right)<\infty
$$


(recall that if $u$ was $\alpha$-harmonic, then $\mu_{n}\left(\mathbb{R}^{d}\right)=u\left(x_{0}\right)$ ). We obtain

$$
u_{n}^{*}(x)=\int_{D_{1 / n}} \frac{G_{D_{1 / n}}(x, \xi)}{G_{D_{1 / n}}\left(x_{0}, \xi\right)} \mu_{n}(d \xi)
$$

The only other property of the function $u$ intervening in the proof of the existence part of the $\Delta^{\alpha / 2}$-Martin representation in [6] is

$$
\lim _{n} \int_{D_{1 / n}^{c}} u(y) d y=0
$$

and it also holds in our case: the $L$-harmonic function $u$ is integrable on $D_{1 / n}^{c}$ for every $n$. The sequence $\left(\mu_{n}\right)$ of simultaneously bounded finite measures with support contained in $\bar{D}$ is tight. We choose a subsequence $\mu_{n_{k}}$ converging to a finite (perhaps zero) measure $\mu$. This choice is common for all $x$. Without loss of generality, we may suppose that $\left(n_{k}\right)$ is a subsequence of $\left(2^{-n}\right)$. The limit measure $\mu$ satisfies

$$
\operatorname{supp}(\mu) \subset \partial D
$$

Exactly as in the proof of the existence part of the $\Delta^{\alpha / 2}-$ Martin representation in [6], we deduce that for all $x \in D$ the limit

$$
\lim _{k} u_{n_{k}}^{*}(x)=u^{*}(x)
$$

exists and

$$
u^{*}(x)=\int_{\partial D} M_{D}(x, Q) d \mu(Q)
$$

Furthermore, in view of (12), for $x \in D_{1 / n}$ and $r>0$ sufficiently small, we have

$$
\nabla u_{n}^{*}(x)=\nabla_{x} \int_{B(x, r)^{c}} P_{B(x, r)}(x, y) u_{n}^{*}(y) d y=\int_{B(x, r)^{c}} \nabla_{x} P_{B(x, r)}(x, y) u_{n}^{*}(y) d y .
$$

By Lemma 1 and (10), we have

$$
\left|\nabla_{x} P_{B(x, r)}(x, y) u_{n}^{*}(y)\right| \leq C \frac{P_{B(x, r)}(x, y)}{r-|x|} u(y)
$$

and by the dominated convergence we get $\nabla u_{n_{k}}^{*}(x) \rightarrow \nabla u^{*}(x)$ as $k \rightarrow \infty$. We also have $\tilde{G}_{D_{1 / n}}(x, w) \nearrow \tilde{G}_{D}(x, w)$. In order to justify the passage with the limit under the integral sign in (23) with $n_{k}$ instead of $n$ we observe that the functions $\tilde{G}_{D_{1 / n_{k}}}(x, w) b(w) \cdot \nabla u_{n_{k}}^{*}(w)$ are uniformly integrable on $D$. Clearly, by Lemma 1 , 
we have $c^{-1} u_{n}^{*}(w) \leq u(w) \leq c u_{n}^{*}(w)$, where $c$ does not depend on $n$, thus $u_{n}^{*}(w) \leq$ $c u^{*}(w)$. By the gradient estimates, we get

$$
\tilde{G}_{D_{1 / n}}(x, w)|b(w)|\left|\nabla u_{n}^{*}(w)\right| \leq \tilde{G}_{D_{1 / n}}(x, w)|b(w)| \frac{u^{*}(w)}{\delta_{D_{1 / n}}(w)},
$$

and the uniform integrability follows from (24), Lemma 1 and Lemma 6. Therefore,

$$
u(x)=u^{*}(x)+\int_{D} \tilde{G}_{D}(x, w) b(w) \cdot \nabla u^{*}(w) d w
$$

which, using (24), becomes

$$
\begin{aligned}
u(x)= & \int_{\partial D} M_{D}(x, Q) d \mu(Q) \\
& +\int_{D} \tilde{G}_{D}(x, w) b(w) \cdot \nabla_{w} \int_{\partial D} M_{D}(w, Q) d \mu(Q) d w .
\end{aligned}
$$

By the gradient estimates and dominated convergence, we also get

$$
\nabla_{w} \int_{\partial D} M_{D}(w, Q) d \mu(Q)=\int_{\partial D} \nabla_{w} M_{D}(w, Q) d \mu(Q), \quad w \in D .
$$

Define a measure $v$ on $\partial D$ by $v(d Q)=l_{D}\left(x_{0}, Q\right) d \mu(Q)$. As the function $Q \rightarrow$ $l_{D}\left(x_{0}, Q\right)$ is continuous positive, the measure $v$ is finite positive on $\partial D$. Using Fubini theorem in (26) and the perturbation formula for $\tilde{M}_{D}$ from Theorem 8 , we obtain

$$
u(x)=\int_{\partial D} \tilde{M}_{D}(x, Q) d v(Q)
$$

Remark 2 We point out that the proof of Theorem 13 is based on the perturbation formula. In fact, the methods used in [6] in order to prove the Martin representation theorem for singular $\alpha$-harmonic functions can not be applied in the present case because the Green function $\tilde{G}_{D}(x, y)$ is not $L$-harmonic on $D \backslash\{x\}$ as a function of $y$.

Corollary 14 (Perturbation formula for singular $L$-harmonic functions) Let $v(x) \geq 0$ be a singular L-harmonic function on $D$ with the Martin representation

$$
v(x)=\int_{\partial D} \tilde{M}_{D}(x, Q) d v(Q), \quad x \in D
$$


Define a singular $\alpha$-harmonic function $v^{*}$ on $D$ by

$$
v^{*}(x)=\int_{\partial D} M_{D}(x, Q) \frac{d v(Q)}{l\left(x_{0}, Q\right)}, \quad x \in D .
$$

Then the following formula holds

$$
v(x)=v^{*}(x)+\int_{D} \tilde{G}_{D}(x, w) b(w) \cdot \nabla v^{*}(w) d w .
$$

Proof Observe that by (16) there exists $\delta>0$ such that

$$
l_{D}\left(x_{0}, Q\right)>\delta>0
$$

for all $Q \in \partial D$. Thus the measure $d \mu(Q)=\frac{d \nu(Q)}{l_{D}\left(x_{0}, Q\right)}$ is finite and the function $v^{*}$ is well defined. By the unicity of the Martin representation and the formula (24), the function $v^{*}$ defined by (28) is the same as the function $v^{*}$ defined by a limit procedure and associated to $v$ in the proof of the Theorem 13. Hence, the formula (25) holds for $v$ and $v^{*}$. It is equivalent to (29).

Corollary 15 Let $v(x) \geq 0$ be a singular L-harmonic function on $D$. The functions $v$ and $v^{*}$ are comparable: there exists $c>0$ such that for all $x \in D$

$$
c^{-1} v^{*}(x) \leq v(x) \leq c v^{*}(x)
$$

Proof We use the Martin representations (27), (28), the Corollary 9 and the fact that $l_{D}\left(x_{0}, Q\right)>\delta>0$ for all $Q \in \partial D$.

\subsection{Perturbation formulas in the diffusion case}

In the present article we exploit the perturbation formulas in the case of the singular operator $L=\Delta^{\alpha / 2}+b \cdot \nabla, 1<\alpha<2$. In this short chapter we make a parenthesis and briefly discuss the case $\alpha=2$ and $d \geq 3$, corresponding to the diffusion operator

$$
L=\frac{1}{2} \Delta+b \cdot \nabla
$$

on $\mathbb{R}^{d}$. The potential theory for such diffusion generators was studied by Cranston and Zhao[23], and more recently by Ifra and Riahi [27], Kim and Song [34] and Luks [38]. Our methods allow to enrich this theory by some new perturbation formulas.

We suppose that $b \in \mathcal{K}_{d}^{1}$ and we assume additionally that $D$ is connected, i.e. it is a domain. Recall that Cranston and Zhao [23] worked under this condition and a complementary second condition $|b|^{2} \in \mathcal{K}_{d-1}^{1}$; Kim and Song [34] suppressed the condition on $|b|^{2}$ and considered signed measures in the place of $b$. 
Proposition 16 Let $L=\frac{1}{2} \Delta+b \cdot \nabla$ with $b \in \mathcal{K}_{d}^{1}$. Then, the following perturbation formula for the L-Green function $\tilde{G}_{D}$ holds if $x, y \in \mathbb{R}^{d}, x \neq y$

$$
\tilde{G}_{D}(x, y)=G_{D}(x, y)+\int_{D} \tilde{G}_{D}(x, z) b(z) \cdot \nabla_{z} G_{D}(z, y) d z .
$$

Proof Note that by [34, Theorem 6.2], we have the estimate

$$
\tilde{G}_{D}(x, y) \leq C|x-y|^{2-d}, \quad x, y \in \mathbb{R}^{d} .
$$

The proof of the Proposition is the same as the proof of [10, Lemma 12] in the case $1<\alpha<2$, with (32) replacing [10, Lemma 7].

Let us mention that a perturbation formula for the $L$-Green function was proposed in [27], but under a restrictive assumption of boundedness of the Kato norm $\|b\|$ of $b$. A simpler direct proof of the estimate (32) without using the precise estimates [34, Theorem 6.2] should be available.

Next we obtain a perturbation formula for the Martin kernel of Laplacians with a gradient perturbation.

Proposition 17 Let $L=\frac{1}{2} \Delta+b \cdot \nabla$ with $b \in \mathcal{K}_{d}^{1}$. Then the following perturbation formula for the L-Martin kernel $\tilde{M}_{D}$ holds if $x \in D$ and $Q \in \partial D$

$$
\tilde{M}_{D}(x, Q)=\frac{1}{l_{D}\left(x_{0}, Q\right)}\left[M_{D}(x, Q)+\int_{D} \tilde{G}_{D}(x, z) b(z) \cdot \nabla_{z} M_{D}(z, Q) d z\right],
$$

where $l_{D}\left(x_{0}, Q\right)$ is a continuous function on $\partial D$, equal

$$
l_{D}\left(x_{0}, Q\right)=M_{D}\left(x_{0}, Q\right)+\int_{D} \tilde{G}_{D}\left(x_{0}, z\right) b(z) \cdot \nabla_{z} M_{D}(z, Q) d z>0 .
$$

Proof We follow the proof of the Theorem 8 in the case $\alpha=2$.

The next perturbation formula concerns the $L$-Poisson kernel $\tilde{P}_{D}(x, Q)$.

Proposition 18 Let $L=\frac{1}{2} \Delta+b \cdot \nabla$ with $b \in \mathcal{K}_{d}^{1}$. Then the following perturbation formula for the L-Poisson kernel $\tilde{P}_{D}$ holds if $x \in D$ and $Q \in \partial D$

$$
\tilde{P}_{D}(x, Q)=P_{D}(x, Q)+\int_{D} \tilde{G}_{D}(x, z) b(z) \cdot \nabla_{z} P_{D}(z, Q) d z .
$$

Proof Observe that by the formula (31) the function $\tilde{G}_{D}$ has the same differentiability properties as the function $G_{D}$. In particular the inner normal derivative $\frac{\partial \tilde{G}_{D}}{\partial n}(x, Q)$ 
exists for $x \in D$ and $Q \in \partial D$. It is known (see [27, page 173]) and possible to prove by the Green formula that

$$
\tilde{P}_{D}(x, Q)=\frac{\partial \tilde{G}_{D}}{\partial n}(x, Q) .
$$

The formula (34) then follows by differentiating of the formula (31) in the direction of the inner normal unit vector $n$. We omit the technical details.

Let us finish this section by some remarks. The formula $\tilde{P}_{D}(x, Q)=\frac{\partial \tilde{G}_{D}}{\partial n}(x, Q)$ implies, like in the Laplacian case, that the $L$-Martin and the $L$-Poisson kernels are related by the formula

$$
\tilde{M}_{D}(x, Q)=\frac{\tilde{P}_{D}(x, Q)}{\tilde{P}_{D}\left(x_{0}, Q\right)}
$$

On the other hand, if we insert the formula $M_{D}(x, Q)=\frac{P_{D}(x, Q)}{P_{D}\left(x_{0}, Q\right)}$ into (34), we obtain using (33)

$$
\tilde{P}_{D}(x, Q)=P_{D}\left(x_{0}, Q\right) l_{D}\left(x_{0}, Q\right) \tilde{M}_{D}(x, Q) .
$$

Evaluating the last equation at $x_{0}$ we obtain a formula for the function $l_{D}\left(x_{0}, Q\right)$ intervening in the perturbation formula (33)

$$
l_{D}\left(x_{0}, Q\right)=\frac{\tilde{P}_{D}\left(x_{0}, Q\right)}{P_{D}\left(x_{0}, Q\right)}
$$

and another proof of the formula (35).

\section{Relative Fatou Theorem for $L$-harmonic functions}

We prove in this section an important boundary property of $L$-harmonic functions: the Relative Fatou Theorem. As in the preceding sections, we consider a nonempty bounded $\mathcal{C}^{1,1}$ open set $D$. Recall the Relative Fatou Theorem in the $\alpha$-stable case. It was proved in [39] for Lipschitz sets $D$.

Theorem 19 Let $g$ and $h$ be two non-negative singular $\alpha$-harmonic functions on $D$, with Martin representations

$$
g(x)=\int_{\partial D} M_{D}(x, Q) d \mu^{(g)}(Q), \quad h(x)=\int_{\partial D} M_{D}(x, Q) d \mu^{(h)}(Q), \quad x \in D .
$$

Then, for $\mu^{(h)}$-almost all $Q \in \partial D$,

$$
\lim _{x \rightarrow Q} \frac{g(x)}{h(x)}=f(x)
$$


where $f$ is the density of the absolute continuous part of $\mu^{(g)}$ in the decomposition $\mu^{(g)}=f d \mu^{(h)}+\mu_{\text {sing }}^{(g)}$ with respect to the measure $\mu^{(h)}$, and $x \rightarrow Q$ non-tangentially.

Our objective in this section is to prove an analogous limit property for non-negative singular $L$-harmonic functions $u$ and $v$ on $D$.

If we denote the integral part of the perturbation formula (29) by

$$
I_{v^{*}}(x)=\int_{D} \tilde{G}_{D}(x, w) b(w) \cdot \nabla v^{*}(w) d w
$$

then we have

$$
u=u^{*}+I_{u^{*}}, \quad v=v^{*}+I_{v^{*}}
$$

where $u^{*}$ and $v^{*}$ are singular $\alpha$-harmonic non-negative functions. We write

$$
\frac{u(x)}{v(x)}=\frac{u^{*}(x)}{v^{*}(x)} \frac{1+\frac{I_{u^{*}(x)}}{u^{*}(x)}}{1+\frac{I_{v^{*}(x)}}{v^{*}(x)}}
$$

The limit boundary behaviors of the quotients $\frac{u(x)}{v(x)}$ and $\frac{u^{*}(x)}{v^{*}(x)}$ will be related if we control the limit behavior of the quotients $\frac{I_{u^{*}}(x)}{u^{*}(x)}$ and $\frac{I_{v^{*}}(x)}{v^{*}(x)}$. Thus we start with discussing the properties of the quotient $\frac{I_{h}(x)}{h(x)}$ for a singular $\alpha$-harmonic non-negative function $h$.

Lemma 20 Let the Martin representation $h(x)=\int_{\partial D} M(x, Q) d \mu^{(h)}(Q)$ for some non-negative finite measure $\mu$ on $\partial D$. Then, if $Q \notin \operatorname{supp}\left(\mu^{(h)}\right)$

$$
\lim _{x \rightarrow Q} h(x)=0
$$

and if $Q \in \operatorname{supp}\left(\mu^{(h)}\right)$ and $x \rightarrow Q$ non-tangentially

$$
\lim _{x \rightarrow Q} h(x)=+\infty .
$$

Proof The limit in the case $Q \notin \operatorname{supp}\left(\mu^{(h)}\right)$ follows easily from the Martin representation of $h$ and the Lebesgue theorem. In the case $Q \in \operatorname{supp}\left(\mu^{(h)}\right)$ we use the following result of $\mathrm{Wu}[49]$.

Let $f$ be a $\Delta$-harmonic function on $D$, corresponding via the Martin representation to a finite measure $\mu=\mu^{(h)}$ on $\partial D$. If $Q \in \operatorname{supp} \mu$, then

$$
\liminf _{x \rightarrow Q} f(x)>0,
$$


provided $x \rightarrow Q$ non-tangentially. We have, on $D$ of class $\mathcal{C}^{1,1}$,

$$
\begin{aligned}
f(x) & =\int_{\partial D} P_{D}^{\Delta}(x, y) \mu(d y) \leq c \int_{\partial D} \frac{\delta_{D}(x)}{|x-y|^{d}} \mu(d y) \\
& =c \delta_{D}(x)^{1-\frac{\alpha}{2}} \int_{\partial D} \frac{\delta_{D}(x)^{\frac{\alpha}{2}}}{|x-y|^{d}} \mu(d y) \leq C \delta_{D}(x)^{1-\frac{\alpha}{2}} \int_{\partial D} M_{D}(x, y) \mu(d y) .
\end{aligned}
$$

Consequently,

$$
h(x) \geq \frac{1}{C} \frac{f(x)}{\delta_{D}(x)^{1-\frac{\alpha}{2}}}
$$

and the second part of the Lemma follows.

Lemma 21 The quotient $\frac{I_{h}(x)}{h(x)}$ is bounded. More exactly, there exists $c>0$ such that

$$
c^{-1} \leq 1+\frac{I_{h}(x)}{h(x)} \leq c .
$$

Proof Observe that by Corollary 15 and the formula (29), the quotient $\frac{I_{v^{*}}(x)}{v^{*}(x)}$ is bounded. More exactly, there exists $c>0$ such that

$$
c^{-1} \leq 1+\frac{I_{v^{*}}(x)}{v^{*}(x)} \leq c .
$$

As the function $l_{D}\left(x_{0}, Q\right)$ is bounded, any singular $\alpha$-harmonic non-negative function $h$ is of the form $v^{*}$ for a singular $L$-harmonic non-negative function $v$.

By (4), if we denote

$$
J_{h}(x)=\int_{D} G_{D}(x, w) b(w) \cdot \nabla h(w) d w
$$

then,

$$
I_{h}(x) \sim J_{h}(x)
$$

In particular, by Lemma 21 , the quotient $J_{h}(x) / h(x)$ is bounded. We prove a much stronger property of this quotient in the following lemma.

Lemma 22 Let $h$ be a non-negative singular $\alpha$-harmonic function on $D$, with the Martin representation $h(x)=\int_{\partial D} M_{D}(x, Q) d \mu^{(h)}(Q)$ for a finite measure $\mu^{(h)}$ on дD. Then, when $Q \in \operatorname{supp}\left(\mu^{(h)}\right)$ and $x \rightarrow Q$ non-tangentially, we have

$$
\lim _{x \rightarrow Q} \frac{J_{h}(x)}{h(x)}=0 .
$$


Proof We will show that $\frac{G_{D}(x, w) h(w)}{h(x) \delta_{D}(w)}$ is uniformly integrable in $x \in D$ against the measure $|b(w)| d w$. Let $\varepsilon>0$. Since $J_{h}(x) / h(x)$ is bounded it suffices to show that there is $\delta>0$ such that

$$
\int_{F} \frac{G_{D}(x, w) h(w)}{h(x) \delta_{D}(w)}|b(w)| d w \leq \varepsilon
$$

provided $\lambda(F)<\delta$. Here, $\lambda$ denotes the Lebesgue measure on $\mathbb{R}^{d}$. First, we note that

$$
\begin{aligned}
\int_{F} & \frac{G_{D}(x, w) h(w)}{h(x) \delta_{D}(w)}|b(w)| d w \\
= & \int_{F} \int_{\partial D} \frac{G_{D}(x, w) M_{D}(w, Q)}{h(x) \delta_{D}(w)} d \mu^{(h)}(Q)|b(w)| d w \\
= & \int_{\partial D} \frac{M_{D}(x, Q)}{h(x)}\left(\int_{F} \frac{G_{D}(x, w) M_{D}(w, Q)}{M_{D}(x, Q) \delta_{D}(w)}|b(w)| d w\right) d \mu^{(h)}(Q) .
\end{aligned}
$$

The function $\frac{G_{D}(x, w) G_{D}(w, y)}{G_{D}(x, y) \delta(w)}$ is uniformly integrable in $x, y \in D$ against $|b(w)| d w$ (see the proof of [10, Lemma 11]). Hence, there exists $\delta>0$ such that for $\lambda(F)<\delta$,

$$
\int_{F} \frac{G_{D}(x, w) G_{D}(w, y)}{G_{D}(x, y) \delta_{D}(w)}|b(w)| d w<\varepsilon, \quad x, y \in D,
$$

and consequently

$$
\begin{aligned}
\int_{F} \frac{G_{D}(x, w) M_{D}(w, Q)}{M_{D}(x, Q) \delta_{D}(w)}|b(w)| d w & =\int_{F} \lim _{D \ni y \rightarrow Q} \frac{G_{D}(x, w) G_{D}(w, y)}{G_{D}(x, y) \delta_{D}(w)}|b(w)| d w \\
& =\lim _{D \ni y \rightarrow Q} \int_{F} \frac{G_{D}(x, w) G_{D}(w, y)}{G_{D}(x, y) \delta_{D}(w)}|b(w)| d w \leq \varepsilon .
\end{aligned}
$$

Now, (38) follows from (39) and Martin representation of $h$. For $Q \in \operatorname{supp} \mu^{(h)}$, $\lim _{D \ni x \rightarrow Q} h(x)=\infty$ from the Lemma 20 . Hence, by uniform integrability,

$$
\begin{aligned}
\lim _{D \ni x \rightarrow Q} \frac{\left|J_{h}(x)\right|}{h(x)} & \leq c \lim _{D \ni x \rightarrow Q} \int_{D} \frac{G_{D}(x, w) h(w)}{h(x) \delta_{D}(w)}|b(w)| d w \\
& =c \int_{D} \lim _{D \ni x \rightarrow Q} \frac{G_{D}(x, w) h(w)}{h(x) \delta_{D}(w)}|b(w)| d w=0 .
\end{aligned}
$$


Now, we return to the Relative Fatou Theorem for $L$-harmonic functions. Let $u$ and $v$ be two non-negative singular $L$-harmonic functions on $D$. By Theorem 13 , they have a Martin representation

$$
u(x)=\int_{\partial D} \tilde{M}_{D}(x, Q) d \mu(Q), \quad v(x)=\int_{\partial D} \tilde{M}_{D}(x, Q) d v(Q), \quad x \in D
$$

where $\mu$ and $v$ are two Borel finite measures concentrated on $\partial D$.

We decompose the measure $\mu$ into its absolutely continuous and singular parts with respect to the measure $v$

$$
d \mu=f d v+d \mu_{\text {sing }}
$$

with a non-negative function $f \in L^{1}(v)$ and $v\left(\operatorname{supp}\left(\mu_{\text {sing }}\right)\right)=0$.

Theorem 23 (Relative Fatou Theorem) For $v$-almost every point $Q \in \partial D$, we have

$$
\lim _{x \rightarrow Q} \frac{u(x)}{v(x)}=f(Q)
$$

when $x \rightarrow Q$ non-tangentially.

Proof We will use the Relative Fatou Theorem for the singular $\alpha$-harmonic functions $u^{*}$ and $v^{*}$ defined according to (28).

Let $Q \in \operatorname{supp}(v) \backslash \operatorname{supp}(\mu)$. Then, if $x \rightarrow Q, v^{*}(x) \rightarrow \infty$ and $u^{*}(x) \rightarrow 0$, so $\lim _{x \rightarrow Q} \frac{u^{*}(x)}{v^{*}(x)}=0$. The formulas (37) and (36) imply that in this case

$$
\lim _{x \rightarrow Q} \frac{u(x)}{v(x)}=0
$$

Let us consider the case $Q \in \operatorname{supp}(\nu) \cap \operatorname{supp}(\mu)$. As

$$
\frac{d \mu(Q)}{l_{D}\left(x_{0}, Q\right)}=f \frac{d \nu(Q)}{l_{D}\left(x_{0}, Q\right)}+\frac{d \mu_{\text {sing }}(Q)}{l_{D}\left(x_{0}, Q\right)}
$$

the Relative Fatou Theorem for the singular $\alpha$-harmonic functions $u^{*}$ and $v^{*}$ says that for $\nu$-almost every point $Q \in \partial D$,

$$
\lim _{x \rightarrow Q} \frac{u^{*}(x)}{v^{*}(x)}=f(Q)
$$

when $x \rightarrow Q$ non-tangentially. The formula (40) then follows by the formula (36) and the Lemma 22.

Acknowledgments We thank Krzysztof Bogdan, Jacek Małecki and Michał Ryznar for discussions about this paper. We are grateful to the anonymous referee for remarks which improved the paper. 
Open Access This article is distributed under the terms of the Creative Commons Attribution License which permits any use, distribution, and reproduction in any medium, provided the original author(s) and the source are credited.

\section{References}

1. Aikawa, H.: Boundary Harnack principle and Martin boundary for a uniform domain. J. Math. Soc. Japan 53(1), 119-145 (2001)

2. Alibaud, N., Imbert, C.: Fractional semi-linear parabolic equations with unbounded data. Trans. Am. Math. Soc. 361, 2527-2566 (2009)

3. Ancona, A.: Principe de Harnack a la frontiere et theoreme de Fatou pour un operateur elliptique dans un domaine lipschitzien. Ann. Inst. Fourier (Grenoble) 28(4), 169-213 (1978)

4. Armitage, D., Gardiner, S.: Classical Potential Theory. Springer, London (2001)

5. Bass, R.F.: Probabilistic Techniques in Analysis. Springer, New York (1995)

6. Bogdan, K.: Representation of $\alpha$-harmonic functions in Lipschitz domains. Hiroshima Math. J. 29(2), 227-243 (1999)

7. Bogdan, K., Byczkowski, T., Kulczycki, T., Ryznar, M., Song, R., Vondracek, Z.: Potential analysis of stable processes and its extensions. In: Graczyk, P., Stos, A. (eds.) Lecture Notes in Mathematics, 1980. Springer, Berlin (2009)

8. Bogdan, K., Dyda, B.: Fatou Relative theorem for harmonic functions of rotation invariant stable processes in smooth domains. Studia Math. 157, 83-96 (2003)

9. Bogdan, K., Jakubowski, T.: Estimates of heat kernel of fractional Laplacian perturbed by gradient operators. Commun. Math. Phys. 271(1), 179-198 (2007)

10. Bogdan, K., Jakubowski, T.: Estimates of the Green function for the fractional Laplacian perturbed by gradient. Potential Anal. 36, 455-481 (2012)

11. Bogdan, K., Kulczycki, T., Kwaśnicki, M.: Estimates and structure of $\alpha$-harmonic functions. Probab. Theory Related Fields 140(3-4), 345-381 (2008)

12. Bogdan, K., Kulczycki, T., Nowak, A.: Gradient estimates for harmonic and q-harmonic functions of symmetric stable processes. Illinois J. Math. 46(2), 541-556 (2002)

13. Borovkov, K., Novikov, A.: On exit times of Levy-driven Ornstein-Uhlenbeck processes. Statist. Probab. Lett. 78(12), 1517-1525 (2008)

14. Brandolese, L., Karch, G.: Far field asymptotics of solutions to convection equation with anomalous diffusion. J. Evol. Equ. 8, 307-326 (2008)

15. Caffarelli, L., Fabes, E., Mortola, S., Salsa, S.: Boundary behavior of nonnegative solutions of elliptic operators in divergence form. Indiana Univ. Math. J. 30, 621-640 (1981)

16. Caffarelli, L., Roquejoffre, J.-M., Sire, Y.: Variational problems for free boundaries for the fractional Laplacian. J. Eur. Math. Soc. 12(5), 1151-1179 (2010)

17. Chen, Z.-Q., Kim, P.: Green function estimates for censored stable processes. Probab. Theory Related Fields 124, 595-610 (2002)

18. Chen, Z.-Q., Kim, P.: Stability of Martin boundary under nonlocal Feynman-Kac perturbations. Probab. Theory Related Fields 128, 525-564 (2004)

19. Chen, Z.-Q., Kim, P., Song, R.: Dirichlet heat kernel estimates for fractional Laplacian with gradient perturbation. Ann. Probab. (2010, to appear)

20. Chen, Z.-Q., Song, R.: Estimates on Green functions and Poisson kernels for symmetric stable processes. Math. Ann. 312(3), 465-501 (1998)

21. Chen, Z.-Q., Song, R.: Martin boundary and integral representation for harmonic functions of symmetric stable processes. J. Funct. Anal. 159, 267-294 (1998)

22. Cranston, M., Fabes, E., Zhao, Z.: Conditional gauge and potential theory for the Schrödinger operator. Trans. Am. Math. Soc. 307(1), 171-194 (1988)

23. Cranston, M., Zhao, Z.: Conditional transformation of drift formula and potential theory for $\frac{1}{2} \Delta+b \cdot \nabla$. Commun. Math. Phys. 112(4), 613-625 (1987)

24. Doob, J.L.: Classical Potential Theory and Its Probabilistic Counterpart. Springer, New York (1984)

25. Fino, A., Karch, G.: Decay of mass for nonlinear equation with fractional Laplacian. Monatshefteur Mathematik 160(4), 375-384 (2010)

26. Hunt, R.A., Wheeden, R.L.: Positive harmonic functions on Lipschitz domains. Trans. Am. Math. Soc. 147, 507-527 (1970) 
27. Ifra, A., Riahi, L.: Estimates of Green functions and harmonic measures for elliptic operators with singular drift terms. Publ. Math. 49, 159-177 (2005)

28. Jakubowski, T.: The estimates for the Green function in Lipschitz domains for the symmetric stable processes. Probab. Math. Stat. 22(2, Acta Univ. Wratislav. No. 2470), 419-441 (2002)

29. Jakubowski, T.: The estimates of the mean first exit time from a ball for the $\alpha$-stable Ornstein-Uhlenbeck processes. Stochastic Process. Appl. 117(10), 1540-1560 (2007)

30. Jakubowski, T.: On Harnack inequality for $\alpha$-stable Ornstein-Uhlenbeck processes. Math. Z. 258(3), 609-628 (2008)

31. Jerison, D.S., Kenig, C.E.: Boundary value problems on Lipschitz domains. In: Studies in Partial Differential Equations. MAA Stud. Math., vol. 23, pp. 1-68. Math. Assoc. America, Washington (1982)

32. Kim, P.: Relative Fatou's theorem for $(-\Delta)^{\alpha / 2}$-harmonic functions in bounded $\kappa$-fat open sets. J. Funct. Anal. 234(1), 70-105 (2006)

33. Kim, P., Lee, Y.-R.: Generalized 3G theorem and application to relativistic stable process on nonsmooth open sets. J. Funct. Anal. 246(1), 113-143 (2007)

34. Kim, P., Song, R.: Two-sided estimates on the density of Brownian motion with singular drift. Illinois J. Math. 50(1-4), 635-688 (2006)

35. Kim, P., Song, R.: Boundary behavior of harmonic functions for truncated stable processes. J. Theor. Probab. 21(2), 287-321 (2008)

36. Kulczycki, T.: Properties of Green function of symmetric stable processes. Probab. Math. Stat. 17(2, Acta Univ. Wratislav. No. 2029), 339-364 (1997)

37. Kunita, H., Watanabe, S.: Markov processes and Martin boundaries, part I. Illinois J. Math. 9, 485-526 (1965)

38. Luks, T.: Hardy spaces for the Laplacian with lower order perturbations. Stud. Math. 204, 39-62 (2011)

39. Michalik, K., Ryznar, M.: Relative Fatou theorem for $\alpha$-harmonic functions in Lipschitz domains. Illinois J. Math. 48(3), 977-998 (2004)

40. Michalik, K., Ryznar, M.: Hardy spaces for $\alpha$-harmonic functions in regular domains. Math. Z. 265(1), 173-186 (2010)

41. Michalik, K., Samotij, K., Martin representation for $\alpha$-harmonic functions, Probab. Math. Stat. 20(1, Acta Univ. Wratislav. No. 2246), 75-91 (2000)

42. Murata, M.: Semismall perturbations in the Martin theory for elliptic equations. Israel J. Math. 102, 29-60 (1997)

43. Novikov, A.A.: Martingales and first-exit times for the Ornstein-Uhlenbeck process with jumps. Theory Probab. Appl. 48, 340-358 (2003)

44. Pinchover, Y.: On positive solutions of second-order elliptic equations, stability results and classification. Duke Math. J. 57, 955-980 (1988)

45. Song, R., Wu, J.-M.: Boundary Harnack principle for symmetric stable processes. J. Funct. Anal. 168, 403-427 (1999)

46. Stein, E.M.: Singular integrals and differentiability properties of functions. Princeton University Press, Princeton (1970)

47. Stein, E.M.: Boundary behavior of holomorphic functions of several complex variables. Princeton University Press, Princeton (1972)

48. Widman, K.O.: On the boundary behavior of solutions to a class of elliptic partial differential equations. Ark. Mat. 6, 485-533 (1966)

49. Wu, J.-M.G.: Comparisons of kernel functions, boundary Harnack principle and relative Fatou theorem on Lipschitz domains. Ann. Inst. Fourier (Grenoble) 28(4), 147-167 (1978) 